\title{
Increasing Skin of Color Publications in the Dermatology Literature: A Call to Action
}

\author{
Candrice R. Heath, MD; Vincent A. DeLeo, MD; Susan C. Taylor, MD
}

\section{PRACTICE POINTS}

- Submitting more articles related to skin of color for peer review and publication will increase educational opportunities.

- Journals that publish skin of color articles play a critical role in reducing educational gaps and ultimately help improve patient care for those with skin of color.

T he US population is becoming more diverse. By 2044, it is predicted that there will be a majority minority population in the United States. ${ }^{1}$ Therefore, it is imperative to continue to develop educational mechanisms for all dermatologists to increase and maintain competency in skin of color dermatology, which will contribute to the achievement of health equity for patients with all skin tones and hair types.

Not only is clinical skin of color education necessary, but diversity, equity, and inclusion (DEI) education for dermatologists also is critical. Clinical examination, ${ }^{2}$ diagnosis, and treatment of skin and hair disorders across the skin of color spectrum with cultural humility is essential to achieve health equity. If trainees, dermatologists, other specialists, and primary care clinicians are not frequently exposed to patients with darker skin tones and coily hair, the nuances in diagnosing and treating these patients must be learned in alternate ways.

To ready the nation's physicians and clinicians to care for the growing diverse population, exposure to more images of dermatologic diseases in those with darker skin tones in journal articles, textbooks, conference lectures, and online dermatology image libraries is necessary to help close the skin of color training and practice gap. ${ }^{3,4}$ The following initiatives demonstrate how Cutis has sought to address these educational gaps and remains committed to improving DEI education in dermatology.

\section{Collaboration With the Skin of Color Society}

The Skin of Color Society (SOCS), which was founded in 2004 by Dr. Susan C. Taylor, is a dermatologic organization with more than 800 members representing 32 countries. Its mission includes promoting awareness and excellence within skin of color dermatology through research, education, and mentorship. The SOCS has utilized strategic partnerships with national and international dermatologists, as well as professional medical organizations and community, industry, and corporate groups, to ultimately ensure that patients with skin of color receive the expert care they deserve..$^{5}$ In 2017, Cutis published the inaugural article in its collaboration with the SOCS, ${ }^{6}$ and more articles, which undergo regular peer review, continue to be published quarterly (https://www.mdedge.com /dermatology/skin-color).

\section{Increase Number of Journal Articles on Skin of Color Topics}

Increasing the number of journal articles on skin of color-related topics needs to be intentional, as it is a tool that has been identified as a necessary part of enhancing awareness and subsequently improving patient care. Wilson et $\mathrm{al}^{7}$ used stringent criteria to review all articles published from January 2018 to October 2020 in 52 dermatology journals for inclusion of topics on skin of color, hair in patients with skin of color, diversity and

Dr. Heath is from the Lewis Katz School of Medicine, Temple University, Philadelphia, Pennsylvania. Dr. DeLeo is from the Keck School of Medicine at the University of Southern California, Los Angeles. Dr. Taylor is from the Perelman School of Medicine, University of Pennsylvania, Philadelphia.

The authors report no conflict of interest.

Correspondence: Candrice R. Heath, MD, 3401 N Broad St, 5OPB, Philadelphia, PA 19140 (Candrice.Heath@tuhs.temple.edu). doi: $10.12788 /$ cutis. 0343 
inclusion, and socioeconomic and health care disparities in the skin of color population. The journals they reviewed included publications based on continents with majority skin of color populations, such as Asia, as well as those with minority skin of color populations, such as Europe. During the study period, the percentage of articles covering skin of color ranged from $2.04 \%$ to $61.8 \%$, with an average of $16.8 \%{ }^{7}$

The total number of Cutis articles published during the study period was 709, with 132 (18.62\%) meeting the investigators' criteria for articles on skin of color; these included case reports in which at least 1 patient with skin of color was featured. ${ }^{7}$ Overall, Cutis ranked 16th of the 52 journals for inclusion of skin of color content. Cutis was one of only a few journals based in North America, a non-skin-of-color-predominant continent, to make the top 16 in this study. ${ }^{7}$

Some of the 132 skin of color articles published in Cutis were the result of the journal's collaboration with the SOCS. Through this collaboration, articles were published on a variety of skin of color topics, including DEI (6), alopecia and hair care (5), dermoscopy/ optical coherence tomography imaging (1), atopic dermatitis (1), cosmetics (1), hidradenitis suppurativa (1), pigmentation (1), rosacea (1), and skin cancer (2). These articles also resulted in a number of podcast discussions (https://www.mdedge.com/podcasts/dermatology -weekly), including one on dealing with DEI, one on pigmentation, and one on dermoscopy/optical coherence tomography imaging. The latter featured the SOCS Scientific Symposium poster winners in 2020.

The number of articles published specifically through Cutis's collaboration with the SOCS accounted for only a small part of the journal's 132 skin of color articles identified in the study by Wilson et al. ${ }^{7}$ We speculate that Cutis's display of intentional commitment to supporting the inclusion of skin of color articles in the journal may in turn encourage its broader readership to submit more skin of color-focused articles for peer review.

Wilson et $\mathrm{al}^{7}$ specifically remarked that "Cutis's [Skin of Color] section in each issue is a promising idea."They also highlighted Clinics in Dermatology for committing an entire issue to skin of color; however, despite this initiative, Clinics in Dermatology still ranked 35th of 52 journals with regard to the overall percentage of skin of color articles published. ${ }^{7}$ This suggests that a journal publishing one special issue on skin of color annually is a helpful addition to the literature, but increasing the number of articles related to skin of color in each journal issue, similar to Cutis, will ultimately result in a higher overall number of skin of color articles in the dermatology literature.

Both Amuzie et $\mathrm{al}^{4}$ and Wilson et $\mathrm{al}^{7}$ concluded that the higher a journal's impact factor, the lower the number of skin of color articles published. However, skin of color articles published in high-impact journals received a higher number of citations than those in other lowerimpact journals. ${ }^{4}$ High-impact journals may use Cutis as a model for increasing the number of skin of color articles they publish, which will have a notable impact on increasing skin of color knowledge and educating dermatologists.

\section{Coverage of Diversity, Equity, and Inclusion}

In another study, Bray et $\mathrm{al}^{8}$ conducted a PubMed search of articles indexed for MEDLINE from January 2008 to July 2019 to quantify the number of articles specifically focused on DEI in a variety of medical specialties. The field of dermatology had the highest number of articles published on DEI (25) compared to the other specialties, including family medicine (23), orthopedic surgery (12), internal medicine (9), general surgery (7), radiology (6), ophthalmology (2), and anesthesiology (2). ${ }^{8}$ However, Wilson et al ${ }^{7}$ found that, out of all the categories of skin of color articles published in dermatology journals during their study period, those focused on DEI made up less than $1 \%$ of the total number of articles. Dermatology is off to a great start compared to other specialties, but there is still more work to do in dermatology for DEI. Cutis's collaboration with the SOCS has resulted in 6 DEI articles published since 2017.

\section{Think Beyond Dermatology Education}

The collaboration between Cutis and the SOCS was established to create a series of articles dedicated to increasing the skin of color dermatology knowledge base of the Cutis readership and beyond; however, increased readership and more citations are needed to amplify the reach of the articles published by these skin of color experts. Cutis's collaboration with SOCS is one mechanism to increase the skin of color literature, but skin of color and DEI articles outside of this collaboration should continue to be published in each issue of Cutis.

The collaboration between SOCS and Cutis was and continues to be a forward-thinking step toward improving skin of color dermatology education, but there is still work to be done across the medical literature with regard to increasing intentional publication of skin of color articles. Nondermatologist clinicians in the Cutis readership benefit from knowledge of skin of color, as all specialties and primary care will see increased patient diversity in their examination rooms.

To further ensure that primary care is not left behind, Cutis has partnered with The Journal of Family Practice to produce a new column called Dx Across the Skin of Color Spectrum (https://www.mdedge.com/dermatology /dx-across-skin-color-spectrum), which is co-published in both journals. ${ }^{9,10}$ These one-page fact sheets highlight images of dermatologic conditions in skin of color as well as images of the same condition in lighter skin, a concept suggested by Cutis Associate Editor, Dr. Candrice R. Heath. The goal of this new column is to increase the accurate diagnosis of dermatologic conditions in skin of color and to highlight health disparities related to a particular condition in an easy-to-understand format. Uniquely, Dr. Heath co-authors this content with family physician Dr. Richard P. Usatine. 


\section{Final Thoughts}

The entire community of medical journals should continue to develop creative ways to educate their readership. Medical professionals stay up-to-date on best practices through journal articles, textbooks, conferences, and even podcasts. Therefore, it is best to incorporate skin of color knowledge throughout all educational programming, particularly through enduring materials such as journal articles. Wilson et $\mathrm{al}^{7}$ suggested that a minimum of $16.8 \%$ of a dermatology journal's articles in each issue should focus on skin of color in addition to special focus issues, as this will work toward more equitable dermatologic care.

Knowledge is only part of the equation; compassionate care with cultural humility is the other part. Publishing scientific facts about biology and structure, diagnosis, and treatment selection in skin of color, as well as committing to lifelong learning about the differences in our patients despite the absence of shared life or cultural experiences, may be the key to truly impacting health equity. ${ }^{11}$ We believe that together we will get there one journal article and one citation at a time.

\section{REFERENCES}

1. Colby SL, Ortman JM. Projections of the size and composition of the U.S. population: 2014 to 2060. United States Census Bureau website. Published March 2015. Accessed August 11, 2021. https://www.census .gov/content/dam/Census/library/publications/2015/demo/p25-1143.pdf
2. Grayson C, Heath C. An approach to examining tightly coiled hair among patients with hair loss in race-discordant patient -physician interactions. JAMA Dermatol. 2021;157:505-506. doi:10.1001 /jamadermatol.2021.0338

3. Alvarado SM, Feng H. Representation of dark skin images of common dermatologic conditions in educational resources: a cross -sectional analysis. J Am Acad Dermatol. 2021;84:1427-1431. doi:10.1016/j.jaad.2020.06.041

4. Amuzie AU, Jia JL, Taylor SC, et al. Skin-of-color article representation in dermatology literature 2009-2019: higher citation counts and opportunities for inclusion [published online March 24, 2021]. I Am Acad Dermatol. doi:10.1016/j.jaad.2021.03.063

5. Learn more about SOCS. Skin of Color Society website. Accessed August 11, 2021. https://skinofcolorsociety.org/about-socs/

6. Subash J, Tull R, McMichael A. Diversity in dermatology: a society devoted to skin of color. Cutis. 2017;99:322-324.

7. Wilson BN, Sun M, Ashbaugh AG, et al. Assessment of skin of color and diversity and inclusion content of dermatologic published literature: an analysis and call to action [published online April 20, 2021]. Int J Womens Dermatol. https://doi.org/10.1016/j .ijwd.2021.04.001

8. Bray JK, McMichael AJ, Huang WW, et al. Publication rates on the topic of racial and ethnic diversity in dermatology versus other specialties. Dermatol Online J. 2020;26:13030/qt094243gp.

9. Heath CR, Usatine R. Atopic dermatitis. Cutis. 2021;107:332. doi:10.12788/cutis.0274

10. Heath CR, Usatine R. Psoriasis. Cutis. 2021;108:56. doi:10.12788 /cutis.0298

11. Jones $N$, Heath CR. Hair at the intersection of dermatology and anthropology: a conversation on race and relationships [published online August 3, 2021]. Pediatr Dermatol. doi:10.1111 /pde.14721 